\title{
Multistatic Radar Measurements of UAVs at X-band and L-band
}

\author{
Piers Beasley, Matthew Ritchie, Hugh Griffiths, William Miceli \\ Department of Electronic and Electrical Engineering \\ University College London \\ London, UK \\ piers.beasley.19@ucl.ac.uk
}

\author{
Michael Inggs, Simon Lewis, Bradley Kahn \\ Department of Electronic and Electrical Engineering \\ Univeristy of Cape Town \\ Rondebosch, South Africa
}

\begin{abstract}
This paper presents analysis of data captured with the NeXtRAD multistatic radar system during a fortnight of experimental trials in December 2019. The trials saw, for the first time, the NeXtRAD system capturing interleaved X-band and L-band measurements of multiple UAVs in simultaneous monostatic and bistatic configurations. Analysis is presented of the UAV's micro-Doppler signatures, permitting a discussion into the challenges some UAV platforms present for reliable detection. Comparisons are also made between $\mathrm{X}$-band and $\mathrm{L}$ band monostatic and bistatic UAV radar backscatter allowing conclusions to be drawn over the benefits of particular radar configurations for aiding UAV detection.
\end{abstract}

Keywords-Radar, Bistatic Radar, Multistatic Radar, Micro Doppler, Drone, UAV, Doppler Signatures.

\section{INTRODUCTION}

As off-the-shelf Unmanned Arial Vehicle (UAV) capabilities increase and their anti-social uses proliferate, methods for detecting these targets becomes ever more crucial. The past decade has seen low cost consumer UAVs become infamous in the media for causing flight delays and, in some cases, the prolonged shutdown of airports. UAVs have also seen use by nefarious organizations as a vehicle for bombings [1] and other criminal activities. One reported case of interest was the assassination attempt on Venezuelan President linked to an almost identical Hexacopter to the one used in these trials [2].

Current challenges are due to existing conventional airsurveillance systems, designed for tracking conventional aircraft, generally performing poorly when tasked with detecting small/medium sized UAVs. This performance issue is attributed to two main factors, a vastly smaller Radar Cross Section (RCS) [3] and the high maneuverability of UAVs. The reduction in RCS presents the radar with both a sensitivity and classification problem. The sensitivity issue is due to the SNR of the UAV backscatter being far lower than that of a conventional aircraft. The classifier issue arises from the similarity in motion and RCS between UAVs and birds. As discussed in [4], unlike commercial aircraft, UAVs have the ability to move at low altitudes, low speeds and hover which presents issues with detection and classification [5]. Standard analysis using range intensity information often yields high false-alarm rates for UAVs due to misclassification of birds and general environmental clutter.

Studying micro-motion, the periodic movement of some structural element of a target or object, is an important avenue for investigation. Some examples of micro-motion are the flapping of a birds wings, the gait of a human's walk or the rotational motion of a UAVs rotor blades [2]. Micro-Doppler is the respective Doppler shift induced by these micro-motions that appear as side bands around the UAV's body bulk Doppler. Micro-Doppler proves a useful metric for classifying between different targets, as presented in [6] [7] [8], where micro-Doppler signatures are proposed as a method for classifying between UAVs and birds. UAV micro-Doppler is now a relatively well documented topic and the mechanisms that produce it have been described extensively in [9] [10]. However, analysis of empirical radar data, detailing the UAV micro-Doppler observed, still continues to be published.

UAV rotor blades produce a peak in RCS when they are perpendicular to the propagation direction of the incident radar pulse. The blade's RCS then reduces as the angle of incidence increases. This peaking in RCS occurs periodically as the rotor spins, producing what is referred to as 'blade flash' [3]. The number of blades per rotor and rotation rate determine the frequency of this periodic flashing. The maximum Doppler shift $\left\{f_{d}\right\}_{\max }$ resulting from blade flash is a product of the radar's RF wavelength $\lambda$, elevation angle referred to the radar $\theta$, length from the tip to blades to the centre $L$ and rotor revolutions per second (RPS) $\Omega$ [9].

$$
\left\{f_{d}\right\}_{\text {max }}=(4 \pi L \Omega / \lambda) \cos \theta
$$

In [3] Rahman et al completed a series of experiments to investigate the micro-Doppler signatures of a range of birds and UAVs using coherent K-Band (24 GHz) and W-band (94 $\mathrm{GHz}$ ) Frequency Modulated Continuous Wave (FMCW) radars. Simultaneous dual band measurements of three different UAVs and four different species of birds were captured. Rahman et al. then use Range Time Intensity (RTI) plots to present time domain data and the Short Time Fourier Transform (STFT) to present the frequency content of the UAVs backscatter as a function of time (often referred to as a spectrogram). The STFT window length simultaneously determines the temporal resolution and frequency resolution. Thus, by selecting a long window duration one can achieve a high Doppler resolution, but the resultant temporal resolution will be reduced. In the results gathered during their trials they observed that the STFT length determined whether UAV blade flash was identifiable or not [4]. When a long STFT length was selected blade flashes were not observed, but instead parallel lines to the UAV's body bulk Doppler appear in the spectrograms. These parallel lines are Helicopter Rotor Modulation (HERM) lines and result from an averaging of blade flash energy across the larger temporal period [3]. This averaging causes a loss in definition of the individual flashes. Further description on the properties of HERM lines is presented later in this report.

This paper is organised as follows. Section II describes the technical details of the NeXtRAD radar system. The experimental radar geometries are then discussed in Section III, followed by a brief overview of the targets used during the experiments in Section IV. Analysis of the radar data is then presented in Section $\mathrm{V}$ and a discussion on observations are summarised in section VI with the conclusions. 


\section{NeXtRAD Multistatic Radar System}

NeXtRAD is a coherent multistatic pulsed radar system consisting of three individual nodes, one transceiver and two identical passive receivers. The radar operates at two bands $8.5 \mathrm{GHz}$ (X-band) and $1.3 \mathrm{GHz}$ (L-band) at a power of $400 \mathrm{~W}$ and $1.6 \mathrm{~kW}$ respectively [11]. Each node has three ADC channels in total, one L-band and two X-band. Dual X-band channels permit simultaneous dual-polarised measurements. Whereas, only single polarised L-band measurements are possible, due to the current system configuration requiring manual polarisation changes [11]. GPS Disciplined Quartz Crystal Oscillators (GPSDOs) are used to provide both phase and temporal synchronisation across the spatially separated nodes. The GPSDOs provide a common $100 \mathrm{MHz}$ reference frequency for phase synchronisation and 1 Pulse Per Second (PPS) signal for temporal synchronisation [12]. A Linear Frequency Modulated chirp pulse with a bandwidth of $50 \mathrm{MHz}$ and pulse duration of $5 \mu \mathrm{S}$ was used for the majority of experiments during the trials. The system can be operated in either single band or dual band mode, where X-band and Lband pulses are sent sequentially in an interleaved manner. PRFs of $1 \mathrm{kHz}$ were used for single-band measurements, and a $500 \mathrm{~Hz}$ per band for dual band measurements. NeXtRAD is a staring radar thus all 3 nodes have identical stationary antenna configurations. A parabolic antenna with azimuth Half Power Beam Width (HPBW) of $13.9^{\circ}$ and elevation HPBW of $12.4^{\circ}$ is used at L-band. A conical horn antenna with azimuth HPBW of $9.1^{\circ}$ and elevation HPBW of $10.4^{\circ}$ was used at X-Band [13]. The transceiver node is quasimonostatic, though Tx and $\mathrm{Rx}$ antennas are again identical.

\section{EXPERIMENTAL GEOMETRIES}

The 2019 trials were conducted on the coast of Simons Town Bay in South Africa. The transceiver was sited on the flat roof of the Institute for Maritime Technology (IMT) building for the duration of the trials, providing an excellent vantage point for observing targets moving across the bay. Bistatic receivers were deployed at three different locations along the coastline during the trials referred to as Lower North (LN), Elsie Bay (EB) and Queens Road (QR). A monostatic configuration on the roof of the IMT was deployed for 3 days, while a bistatic and a multistatic configuration was deployed for 5 days and 1 day respectively. The most regularly used bistatic node location was QR, visible at the bottom of Figure 1. This provided a baseline of between $318-375 \mathrm{~m}$ with the Transceiver (variance due to the location of bistatic node along the road). Since the NeXtRAD system is a staring radar, in this configuration both nodes were directed at either the Roman Rock Lighthouse (RR) or Noah's Ark Rock (NA). These geometries provided bistatic distances of approximately $3950 \mathrm{~m}$ and $1650 \mathrm{~m}$ respectively and bistatic angles of approximately $51^{\circ}$ and $82^{\circ}$ respectively (approximate values in the view of variation in bistatic node location). The multistatic configuration used on the 12th Dec sited a bistatic node at both EB and LN, locations visible in Figure 1. These locations provided baselines of $4028 \mathrm{~m}$ and $2635 \mathrm{~m}$ respectively from the transceiver on the IMT roof.

\section{TARGETS}

Three cooperative targets were used during the trials, two UAVs and a small fishing boat sized experimental watercraft. The larger of the UAV platforms was a DJI Matrice 600 Pro Hexacopter, an enterprise specification UAV primarily used

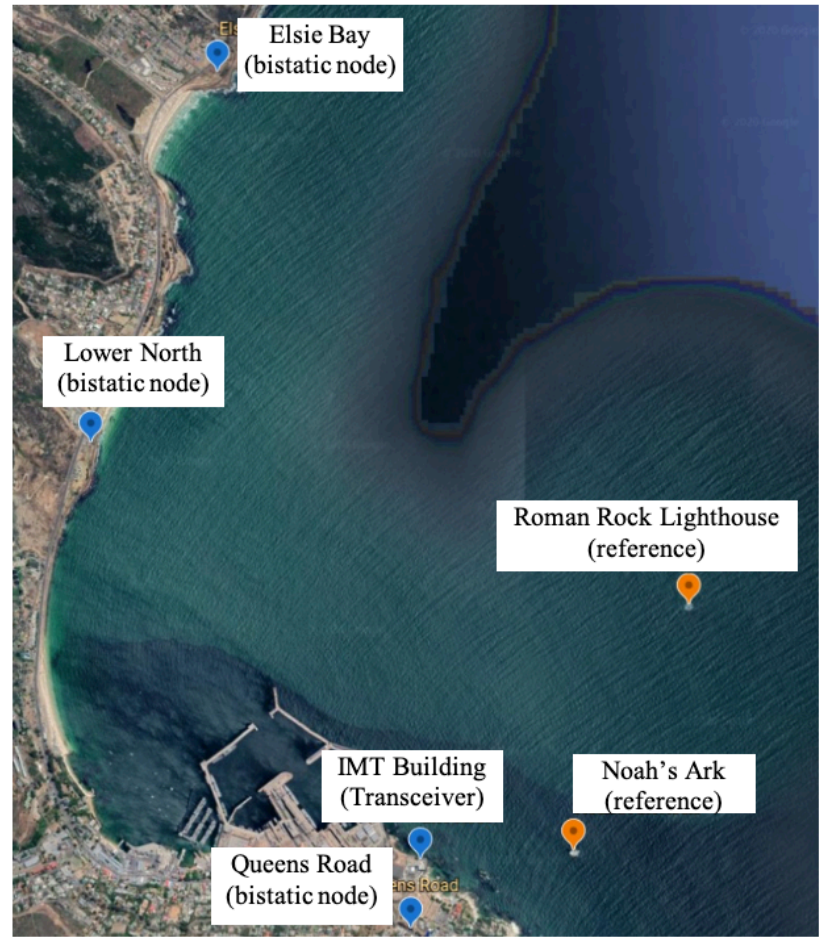

Figure 1-Map of the experimental area. Labelled with deployment locations of the NeXtRAD radar nodes along with stationary reference points visible in radar captures.

by industry, see Figure 2. The Hexacopter has a diagonal span of $1668 \mathrm{~mm}$ (including propellers), a height of $553 \mathrm{~mm}$ (with landing gear retracted) and weight of $10 \mathrm{~kg}$. Of note, the frame is predominantly constructed of carbon fibre tube and plating, which provides strong reflections to the electromagnetic radar pulses in comparison to a similar plastic framed construction. The second, smaller UAV, was a DJI Phantom 4 Quadcopter, shown in Figure 2, a consumer drone chiefly operated by hobbyists. The Quadcopter has a diagonal span of $350 \mathrm{~mm}$ (excluding propellers), height of $196 \mathrm{~mm}$ and weight of 1.39 $\mathrm{kg}$. Unlike the Hexacopter, the Quadcopter has fixed landing gear and a lightweight plastic shell construction.

GPS way-point flightpaths were provided to the UAV pilots before take-off to ensure that the UAVs would manoeuvre within the beam of the radar during the trials. Both UAVs also logged their GPS coordinates every tenth of a second. These GPS logs were subsequently used to provide a ground truth when analysing radar data, permitting an extra factor of certainty to what was concluded as being observed. A plot of the GPS flightpaths for a set of experiments utilizing two UAVs can be seen in Figure 3. The flightpaths instructed each UAV to fly in a circular motion between 12 waypoints at two spatially separated locations between the IMT and Roman Rock. The UAVs were instructed to hover for 5 seconds at each waypoint before proceeding to the next. A graph of dual
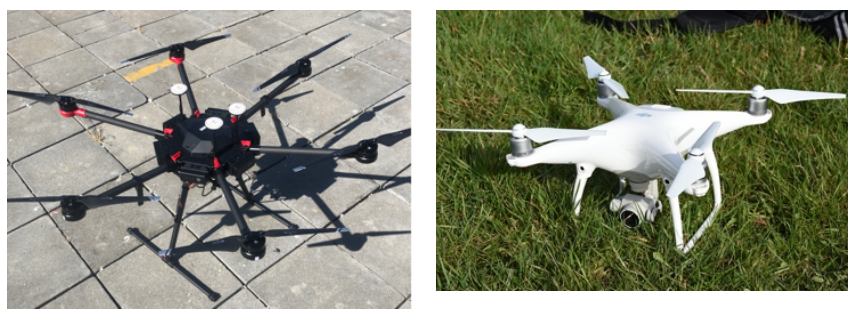

Figure 2-Photos of the two UAVs used during the 2019 NeXtRAD experiments. DJI Matrice 600 Pro Hexacopter (left); DJI Phantom 4 Quadcopter (right). 
path monostatic range and bistatic range against time for both UAVs is presented in Figure 4. The bistatic node was sited on the Queens Road during this experiment. One can observe that the circular motion translates to a sinusoidal change in range when only radial range information is available to the radar.

Sea Lab, a small fishing boat sized experimental watercraft owned by the IMT was the third cooperative target utilized. This target was used in the multistatic configuration on the 12th Dec and maneuvered between EB and LN. In addition, a variety of non-cooperative targets were often present during the trials, including a range of Kayaks, Sailing Boats, Power Boats and birds.

\section{RADAR DATA ANALYSIS}

In total, 419 measurements were taken during the fortnight of trials, accounting for $544 \mathrm{~GB}$ of radar data. This data has been reviewed and the best captures identified. The data presented in this paper are focused on measurements of UAVs, however a large amount data are available that would provide good basis for research into sea clutter and bird microDoppler. Analysis into the radar signatures of the two UAVs has been completed in both the time and frequency domain. Analysis in the time domain was performed after pulse compression where all values were normalized to a peak of zero decibels before being presented in Range Time Intensity (RTI) plots with a dynamic range of $50 \mathrm{~dB}$. The UAV GPS logs discussed in the previous section were superimposed on the RTI plots to aid analysis. Figure 5 is an example of an Lband measurement RTI plot for the Hexacopter's movements during a 90 second capture. The GPS ground truth, overlaid as a solid black line, shows strong correlation with the observed RTI track of the Hexacopter. The sinusoidal like tracks centered around the Hexacopter's path are birds swarming the UAV, a common occurrence during the trials. Frequency analysis was conducted using the Short Time Fourier

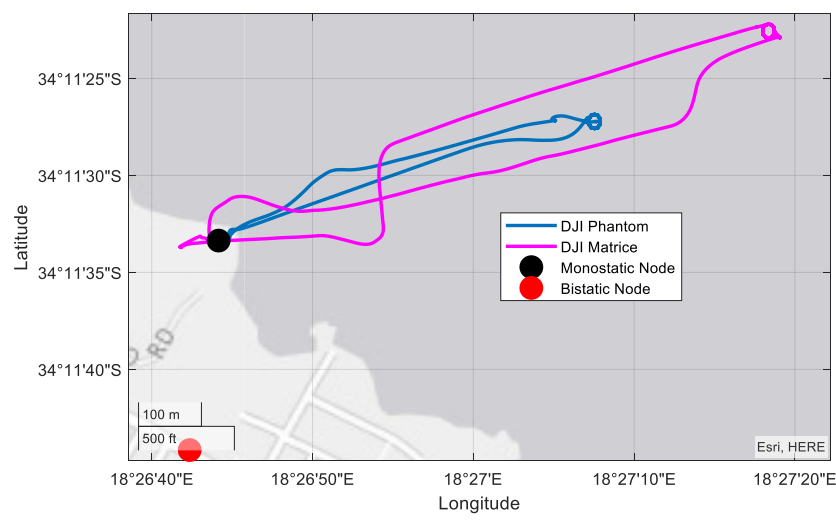

Figure 3 - An example of the UAVs flightpaths, overlaid on map of Simon's Town bay. Ground truth data provided by each UAV's GPS log.

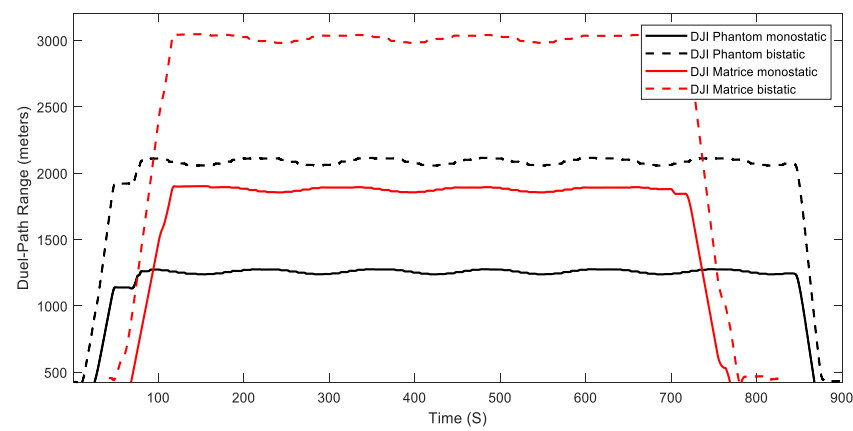

Figure 4-Plot of UAVs monostatic and bistatic ranges for a period of experiments derived from UAV's GPS logs.

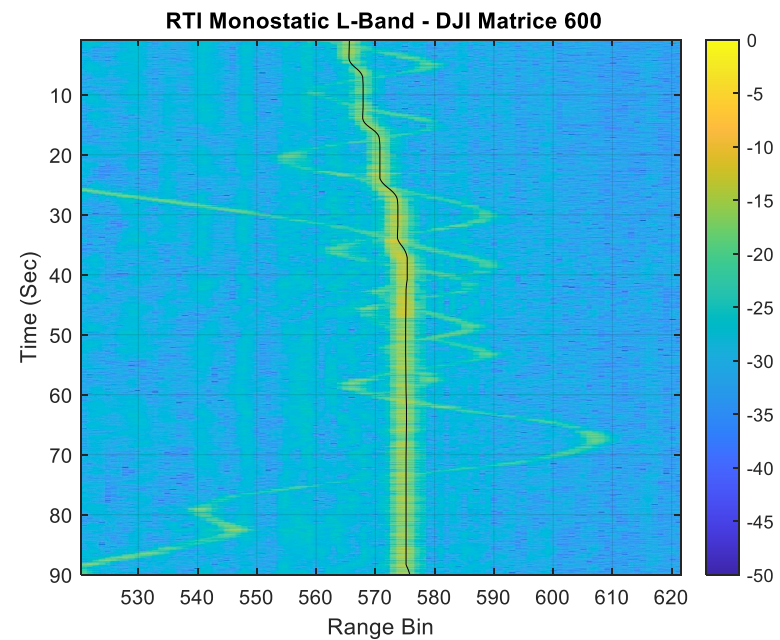

Figure 5 - Monostatic L-band RTI plot of Hexacopter track. Overlaid with GPS ground truth data, visible as thin black line.

Transform (STFT), where a window size of 512 samples, 0.512 seconds, was found to provide the best detail. An overlap of $90 \%$ was also used to remove granularity by smoothing the result of the STFT. The STFT was conducted on a coherent sum of the 5 adjacent range bins either side of the UAVs position (determined using the GPS ground truth). The result of the STFT was first normalized to a peak of zero decibels, before a limit was then imposed on the dynamic range. In most cases $20 \mathrm{~dB}$ of dynamic range was adequate to include UAV micro-Doppler and thus enable analysis.

Some preliminary calculations have been completed using the specifications of both the radar and UAVs to predict the expected micro-Doppler features will be observed. The maximum predicted Doppler shifts produced by the UAVs rotors are calculated using equation 1, assuming an elevation angle of $0^{\circ}$, and tabulated in Table I. The unambiguous Doppler frequency limit of a pulse Doppler radar is a product of the radars PRF. The maximum PRF used during the NeXtRAD trials was $1 \mathrm{kHz}$, translating to unambiguous Doppler frequency limit of $\pm 500 \mathrm{~Hz}$. By comparing the maximum Doppler frequencies in Table I to the unambiguous Doppler frequency limit of the NeXtRAD radar configuration used, one can determine that aliasing will occur of the UAVs micro-Doppler. It is also worth commenting on at this stage what micro-Doppler is expected to be visible in the trials data. It is explained in [14] that the STFT window duration must be small enough in order to not cause loss of temporal features such as blade flash. Note the PRF determines the maximum theoretical temporal resolution (as the higher PRFs permit shorter STFT window durations for the same spectral resolution). In [3], blade flash is observed of a DJI Phantom UAV using a $94 \mathrm{GHz}$ radar with a PRF of $50 \mathrm{kHz}$ and STFT length of 512 (10.2 ms STFT duration). However, when a lower PRF is used in [14] and [3], blade flash is not observed, and instead HERM lines appear in [14]. In this paper, 512 sample STFTs were used when analyzing UAV microDoppler, which results in $0.512 \mathrm{~s}$ and $1.024 \mathrm{~s}$ STFT window

TABLE I. MAXIMUM PREDicted UAV Micro-DOPPLER SHIFTS

\begin{tabular}{|l|c|c|c|c|}
\hline \multirow{2}{*}{ UAV } & \multicolumn{2}{|c|}{$\begin{array}{c}\text { Micro-Doppler of } \\
\text { Hovering UAV }\end{array}$} & \multicolumn{2}{c|}{$\begin{array}{c}\text { Micro-Doppler of UAV } \\
\text { Moving at Max Speed }\end{array}$} \\
\cline { 2 - 5 } & L-Band & X-Band & L-Band & X-Band \\
\hline DJI M600 Pro & $629 \mathrm{~Hz}$ & $4.28 \mathrm{kHz}$ & $1.34 \mathrm{kHz}$ & $9.14 \mathrm{kHz}$ \\
\hline DJI Phantom 4 & $629 \mathrm{~Hz}$ & $4.28 \mathrm{kHz}$ & $943 \mathrm{~Hz}$ & $6.41 \mathrm{kHz}$ \\
\hline
\end{tabular}


durations for single and dual band measurements respectively. Given these long STFT window durations the presence of blade flash can be excluded at this stage, instead one could reasonably expect to observe HERM lines in their place.

\section{A. L-Band Measurement of DJI M600 Pro and Phantom 4}

An L-band measurement was conducted with both the Hexacopter and Quadcopter maneuvering within the radar beam. The monostatic and bistatic STFT results for the 90 second measurement of the Hexacopter are displayed in Figure 6. The associated monostatic RTI plot was presented previously in Figure 5, where the Hexacopter can be seen moving between 930 and 947 metres in range with an average SNR of 20dB. Distinct HERM lines are visible about multiples of $\pm 100 \mathrm{~Hz}$ from the UAV body's bulk Doppler. These HERM lines were calculated to be on average $-7 \mathrm{~dB}$ lower in power than the UAV body's bulk Doppler.

Figure 7 is the monostatic and bistatic range limited RTI plots of the Quadcopter. In the monostatic RTI plot the path of the Quadcopter is almost entirely masked by interference. This interference is an artefact introduced by the radar's Lband amplifier and not a product of the environment it is sampling. The bistatic capture does not experience the same masking and the track of the Quadcopter is clearly visible. The Quadcopter manoeuvres at a monostatic range of 621-638 metres and bistatic range of 1012-1049 metres. An average SNR of $10 \mathrm{~dB}$ was observed for the bistatic capture of quadcopter (due to the amplifier interference a monostatic SNR was not attainable). Figure 8 is a spectrogram of the monostatic capture focused on the range bins where the Quadcopter was manoeuvring. Doppler shifts are visible at $\pm 50 \mathrm{~Hz}$, though these have been proven to be present in range bins without UAVs and therefore treated as artefacts introduced by the radar. Consequently, it was concluded that the Quadcopter micro-Doppler was not visible above the noise floor. Flashes of Doppler attributed to birds intersecting the path of the quadcopter are however visible. These flashes corelate with the time instants where bird tracks intersect the UAV track, visible in Figure 7. Figure 9 compares the
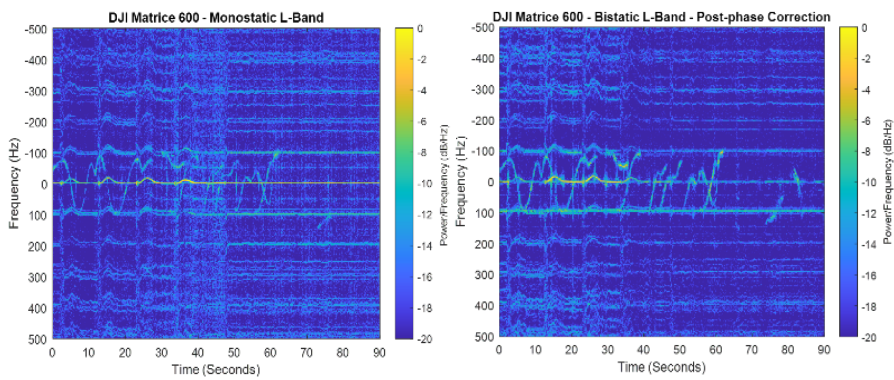

Figure 6-Micro-Doppler signatures of Hexacopter at L-band. Monostatic capture (left); Bistatic capture (right).
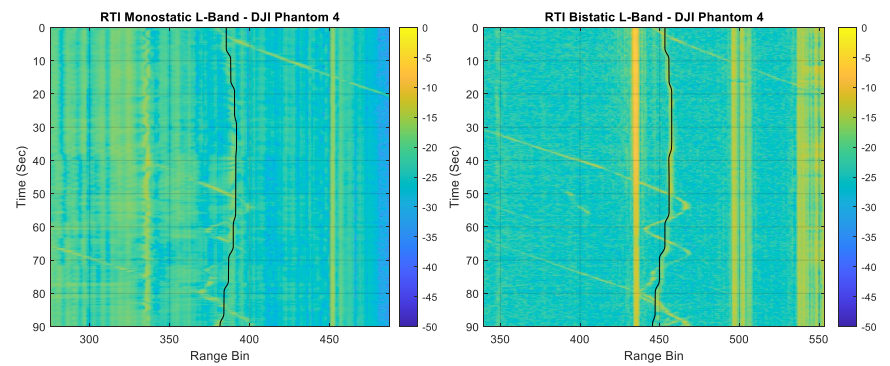

Figure 7 - L-band RTI Plots for track of Quadcopter. Monostatic capture (left); Bistatic capture (right).

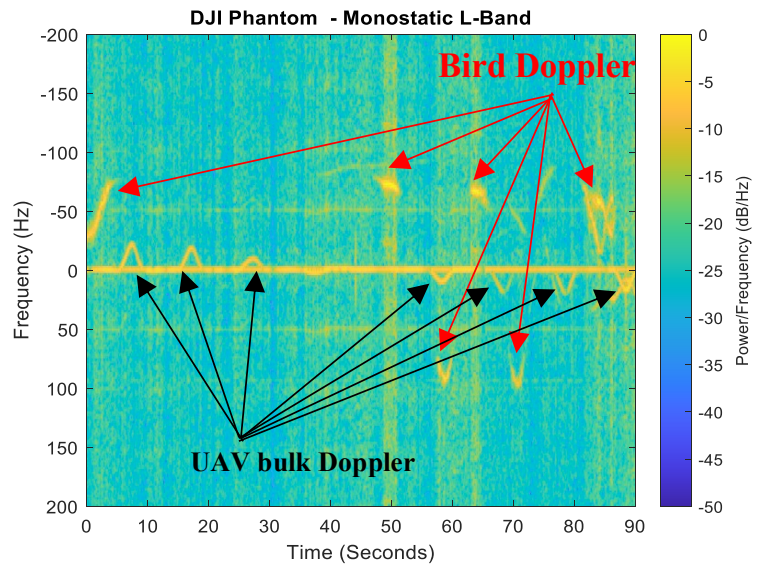

Figure 8 - Spectrogram plot of Quadcopter's track. Quadcopter's bulk Doppler is clearly visible; however, no micro-Doppler is distinguishable above noise.
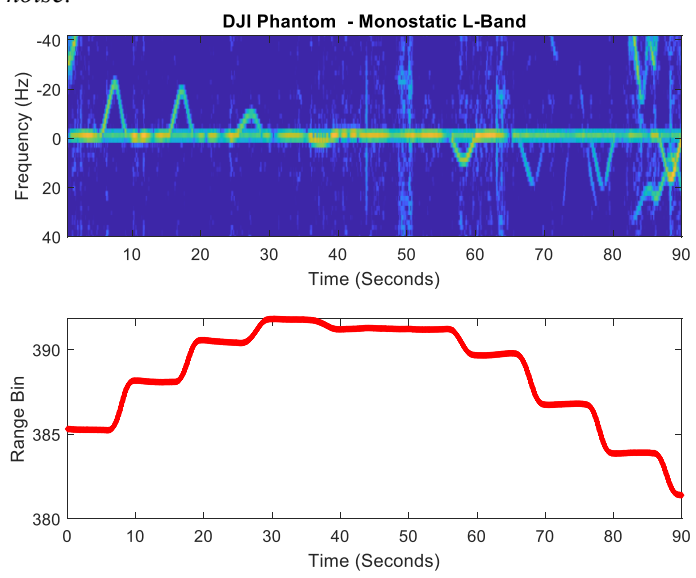

Figure 9 - Spectrogram showing Quadcopter's bulk Doppler shift during 90 s capture (top); Quadcopter monostatic range-over-time derived from UAV GPS log for the same capture 90s capture (bottom).

Quadcopter's bulk Doppler shift to a plot of its monostatic range over time (derived from the UAV GPS log). From comparison of the plots, it is clear that the UAVs bulk Doppler shifts strongly correlate with its change in monostatic range.

\section{B. Co-polarised and Cross-polerised Measurements}

As discussed in Section II the NeXtRAD system is capable of capturing dual polarised X-band measurements, or fully polarimetric measurements when $\mathrm{HH} \mathrm{HV}$ and $\mathrm{VV}$ VH are captured on alternating pulses [11]. During the trials the radar was used with a Horizonal transmission polarization to aid detection of the UAV's propeller micro-Doppler. Therefore, $\mathrm{HH}$ and $\mathrm{HV}$ polarimetric measurements were captured during the trials. A comparison between $\mathrm{HH}$ and $\mathrm{HV}$ monostatic radar measurements of the Hexacopter has been completed in both the time and frequency domain. During this measurement the UAV maneuvered between 933 and 947 metres. The first observable difference between HH and HV captures was the Hexacopter's RTI track SNR. The HV RTI track was $-3.1 \mathrm{~dB}$ the power of the HH RTI capture, which was on average 5.6dB. This could be expected due to the orthogonality of polarization between transmitter and receiver in the $\mathrm{HV}$ capture. The respective $\mathrm{HH}$ and $\mathrm{HV}$ spectrograms for a 20 second segment of the measurement are shown in Figure 10. For the HH capture HERM lines are clearly visible above the noise floor and were calculated to be on average $-11.2 \mathrm{~dB}$ lower in power than the UAV's bulk Doppler. In contrast, the HV capture's spectrogram exhibits far less defined HERM 
TABLE II. X-BAND POLARMETRIC REsults OF HeXacopter

\begin{tabular}{|l|c|c|}
\hline Polarisation & $\begin{array}{c}\text { SNR of UAV in RTI } \\
\text { (time domain) }\end{array}$ & $\begin{array}{c}\text { HERM to UAV bulk } \\
\text { doppler relative power }\end{array}$ \\
\hline Co-pol & $5.6 \mathrm{~dB}$ & $-11.2 \mathrm{~dB}$ \\
\hline Cross-pol & $2.5 \mathrm{~dB}$ & $-7.4 \mathrm{~dB}$ \\
\hline
\end{tabular}
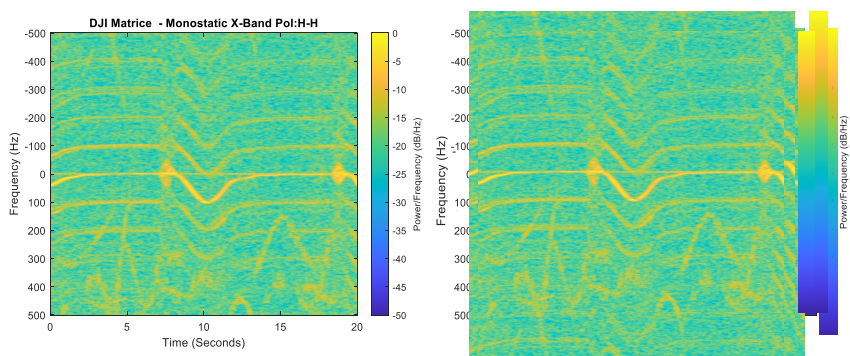

Figure 10-Micro-Doppler signatures of Hexacopter captured during Polarimetric X-band measurement. HH Polarisation (left); HV

Polarisation (right).

lines, though these HERM lines were calculated to be only $-7.4 \mathrm{~dB}$ lower than the UAV's bulk Doppler. However, as visually clear in the two spectrograms the noise floor is far higher in the HV capture, making extracting HERM lines from the HV capture challenging. Therefore, the relative power between the UAV's bulk Doppler and HERM lines should not be used as the sole metric for reporting SNR of HERM lines.

\section{Interleaved Dual-Band Meaasurments}

The NeXtRAD system captured interleaved X-band and L-band measurements for the first time during the 2019 trials. This allowed for virtually simultaneous temporal comparison between X-band and L-band radar measurements. A dualband $\mathrm{HH}$ polarization measurement was taken of the Hexacopter, with a bistatic node located on the Queens Road providing a baseline of 329 metres. Interleaving X-band and L-band effectively halves the PRF, from $1 \mathrm{kHz}$ to $500 \mathrm{~Hz}$ per frequency. This reduction in PRF reduces the maximum unambiguous Doppler frequency from $\pm 500 \mathrm{~Hz}$ to $\pm 250 \mathrm{~Hz}$. Figure 11 contains spectrograms for X-band and L-band measurements at each node. In both monostatic captures HERM lines are visible up until the point in which the UAV enters the same range bins as Noah's Ark rock (an 80-100 metre wide flat-topped rock that protrudes out the water by approximately 10 metres). At this point the returns from the Hexacopter are masked by the high-power returns from the stationary rock. An unexpected observation is the appearance of what look like HERM lines at $\pm 50 \mathrm{~Hz}$ in the monostatic
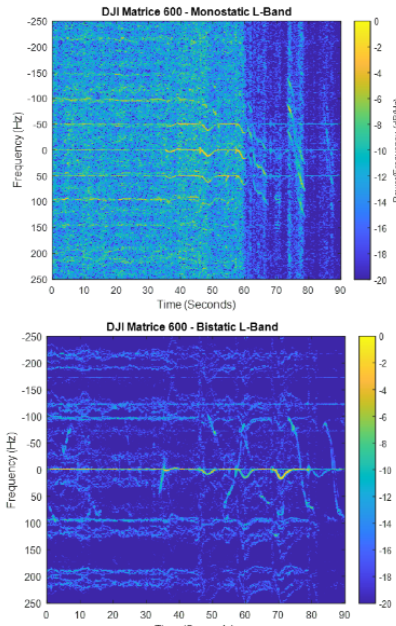
Figure 11
Interleaved Dual Band measurement. L-band monostatic signature (top left); $X$-band monostatic signature (top right); L-band bistatic signature (bottom left); $X$-band bistatic signature (bottom right). spectrograms. These HERM lines are not present in the bistatic captures and in all previous measurements of the Hexacopter HERM lines have appeared at multiples of \pm 100 $\mathrm{Hz}$ from the UAV bulk Doppler. After further investigation, these high power $\pm 50 \mathrm{~Hz}$ Doppler shifts were found to exist at ranges in which there was no Hexacopter present. Therefore, they can be discounted as interference introduced by the radar that has inter-modulated with the UAV's bulk Doppler in the receiver. This conclusion is further confirmed, when during the last 30 seconds of the monostatic captures the $\pm 50 \mathrm{~Hz}$ shifts are still present, even after the bulk Doppler of the UAV has been masked by high power ground clutter.

In both monostatic measurements the HERM lines are far less distinguishable from the interference than their bistatic counterparts. The bistatic captures show a spread of HERM lines around $\pm 100 \mathrm{~Hz}$ and $\pm 200 \mathrm{~Hz}$. This spread of Doppler shifts was not observed in previous captures and is likely the result of under sampling the HERM lines at $300 \mathrm{~Hz}$ and 400 $\mathrm{Hz}$. This under sampling will cause the two HERM lines to fold in at approximately $200 \mathrm{~Hz}$ and $100 \mathrm{~Hz}$ respectfully, resulting in what looks like far less defined HERM at these frequencies. The relative power between HERM lines and the UAV bulk Doppler was observed to be $-9.5 \mathrm{~dB}$ for L-band measurements and $-13.5 \mathrm{~dB}$ for X-band measurements. As expected considerably larger Doppler-shifts were observed in $\mathrm{X}$-band measurements for the same target movements than in L-band. This is due to the magnitude of the Doppler shift being a product of the radar's transmit frequency. The Doppler shift at X-band $(8.5 \mathrm{GHz})$ should be $\sim 6.5$ times greater than observed at L-band $(1.3 \mathrm{GHz})$.

\section{L-band Measurement of Bird}

To provide contrast to the micro-Doppler signatures of UAVs presented in the previous sub-sections, a spectrogram for a short period of flight of a seagull is presented in Figure 12. Capturing long duration measurements of birds is a relatively challenging task due to their non-cooperative nature. This spectrogram was for an L-band capture at a PRF of $1 \mathrm{kHz}$. The variation of Doppler observed is due to the bird accelerating and decelerating as it flaps its wings and glides. Unlike in the UAV equivalent spectrograms very little microDoppler is visible and the characteristic UAV HERM lines are not present, as would be expected.

\section{DISCUSSION}

In Section V (A) the detectability of the two UAVs was analyzed. It was observed that the Hexacopter's SNR was on average $6 \mathrm{~dB}$ higher than the Quadcopter when manoeuvering at a range $50 \%$ further. This result shows that the RCS of the Hexacopter is considerably larger than the Quadcopter. It is thought that this finding is not solely due to the obvious difference in physical size between the two UAVs, but also the material from which the two UAVs are fabricated. The

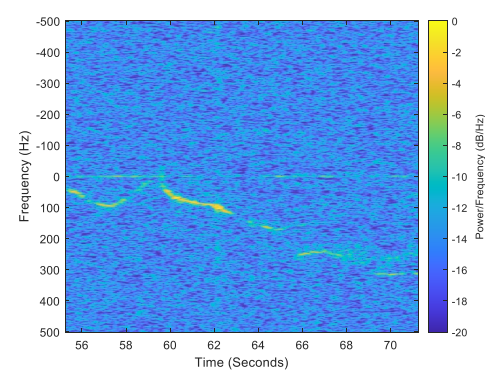

Figure 12 - Micro-Doppler signature of Seagull captured during L-band measurement. 
carbon fiber frame of the Hexacopter will reflect far more electromagnetic energy than the plastic construction of the Quadcopter resulting in it being considerably easier to detect. In regard to the micro-Doppler signatures of the two UAVs, the Hexacopter exhibited HERM lines at multiples of $\pm 100 \mathrm{~Hz}$ and powers of $-7 \mathrm{~dB}$ relative to the UAV's bulk Doppler. Whereas in comparison, no micro-Doppler was visible in spectrograms of the Quadcopter. It has been previously reported that the micro-Doppler produced by the propellers of the same Quadcopter is 20-40dB lower than Quadcopter's bulk Doppler [7] [3]. Thus, an SNR of at least 30-40dB is required for the micro-Doppler of the propellers to be visible above the noise floor. An SNR of just 10dB was observed for the Quadcopter in Section V (A) thus no micro-Doppler was detectable above the noise floor in spectrograms. The propeller blades of the Hexacopter are also fabricated of a composite including carbon fibre which, as previously mentioned, will reflect considerably more radar energy compared to the plastic rotors of the Quadcopter.

The polarimetric measurement in Section V (B) clearly illustrates the difference in return power between co-polarized $(\mathrm{HH})$ and cross-polarized (HV) measurements. It was shown that the Hexacopter's track SNR was approximately $3 \mathrm{~dB}$ lower for the cross-polarized measurement than the copolarized measurement. Comparison between the relative power of the micro-Doppler to the bulk Doppler of the Hexacopter showed that HERM lines in the cross-polar measurement were higher in relative power than in the copolarized measurement. Though, the noise floor was considerably higher in the cross-polarized spectrogram making the HERM lines far less defined. From this we concluded that the relative power between the UAV bulk Doppler and HERM lines is not always appropriate as a sole measure for reporting HERM line power.

The interleaved measurement in Section V (C) allowed direct comparison between X-band and L-band Hexacopter backscatter. An initial observation was the larger magnitude bulk Doppler shift in the X-band capture, which was explained to be the result of the higher transmit frequency. However, what is of notable interest is that the micro-Doppler HERM lines produced by the micro-motion of the Hexacopter propellers. These HERM lines are located at multiples of \pm 100 $\mathrm{Hz}$ from the UAVs bulk Doppler in both X-band and L-band spectrograms. This shows that the frequency spacing of the HERM lines are independent of the transmission frequency of the radar, a theory similarly discussed on a paper examining Jet Engine Modulation phenomena [15]. Instead the HERM line spacing is a product of the propeller rotational rates and number of blades per propeller, as concluded in [14]. The Hexacopter has two blades per propeller and propeller rotational rates of approximately $45-50 \mathrm{~Hz}$ when hovering, thus HERM lines should appear with spacing of roughly $100 \mathrm{~Hz}$ [14], which agrees with what was observed in the trials results presented in this paper.

In conclusion, this report has presented some new empirical results gathered by the NeXtRAD radar system for both Hexacopter and Quadcopter backscatter at X-band and L-band. The considerable challenges posed in capturing the micro-Doppler of the smaller Quadcopter has been discussed, potentially prompting other methods of discrimination to be investigated. The different UAV micro-Doppler artefacts observable by different radars have been discussed also, and reasonable predictions made on what the capabilities of the
NeXtRAD system are in its current configuration. It is clear that UAV micro-Doppler provides a good method for distinguishing between UAVs and other similar size RCS targets such as birds, provided a high enough SNR is achievable.

\section{ACKNOWLEDGMENT}

The authors would like to acknowledge the work done by Francesco Fioranelli, Riccardo Palama, Stephan Sandenbergh and the many others that were instrumental in the design and build of the NeXtRAD system. Additionally, the Office of Naval Research Global (USA), the Institution of Engineering and Technology, FFI Norway and the IMT (South Africa) for the funding provided for the NeXtRAD project. The trials were a collaborative effort between the University of Cape Town (UCT) and University College London (UCL). Michael Inggs, Simon Lewis, Bradley Kahn and Harry Mafukidze were present from UCT. Hugh Griffins, Matthew Ritchie, William Miceli and Piers Beasley were present from UCL. Adrian Stevens, Lance Clayton and multiple other staff from the IMT were present to provide vital support to the trials also. Piers Beasley would like to personally acknowledge the UK Defense Science and Technology Laboratory (DSTL) who continue to provide $\mathrm{PhD}$ sponsorship for his research into active and passive multistatic radar.

\section{REFERENCES}

[1] M. Vaccaro, "The Phantom Menace: A Security Overview of the Phantom 3 Standard," ProQuest Dissertations Publishing, New York, USA, 2017.

[2] A. E. M. Sudhakar, "The Behavior of sUASs under Explosive Loading Conditions and Implications for Safe Operating Procedures," ProQuest Dissertations and Theses, Missouri, USA, 2019.

[3] S. Rahman and D. A. Robertson, "Radar micro-Doppler signatures of drones and birds at K-band and W-band," Scientific Reports, vol. 8, pp. 1-11, 26 November 2018.

[4] R. I. Harmanny, J. J. de Wit and G. Prémel Cabic, "Radar micro-Doppler feature extraction using the spectrogram and the cepstrogram," in 2014 11th European Radar Conference, Rome, Italy, 2014.

[5] M. Lü, J. Yi, X. Wan and Y. Liu, "Experimental Research of Drone Monitoring Using Multi-static Passive Radar," The Journal of Engineering 2019, vol. 2019, no. 20, p. 6795 - 6798, 11 July 2019.

[6] D. Tahmoush, "Detection of small UAV helicopters using micro-Doppler," in Radar Sensor Technology XVIII, Baltimore, USA, 2014.

[7] M. Ritchie, F. Fioranelli, H. Griffiths and B. Torvik, "Monostatic and bistatic radar measurements of birds and micro-drone," in 2016 IEEE Radar Conference, Philadelphia, USA, 2016.

[8] S. Rahman and D. A. Robertson, "Millimeter-wave radar micro-Doppler feature extraction of consumer drones and birds for target discrimination," in Radar Sensor Technology XXIII, Baltimore, Maryland, USA, 2019.

[9] V. C. Chen, The Micro-Doppler Effect in Radar, Norwood, MA: Artech House, 2019.

[10] V. C. Chen, H. Wechsler and S. S. Ho, "Analysis of micro-Doppler signatures," IEE Proceedings-Radar Sonar And Navigation, vol. 150, no. 4, pp. 271-276, August 2003.

[11] M. R. Inggs, S. Lewis, R. Palama, M. A. Ritchie and H. Griffiths, "Report on the 2018 Trials of the Multistatic NeXtRAD Dual Band Polarimetric Radar," in 2019 IEEE Radar Conference (RadarConf), Boston, USA, 2019.

[12] M. R. Inggs, S. Lewis, H. Griffiths, F. Fioranelli, M. Ritchie and K. Woodbridge, "Multistatic Radar: System Requirements and Experimental Validation," in 2014 International Radar Conference, Lille, France, 2015.

[13] S. Alhuwaimel, S. Coetzee, P. Cheng, D. Du Plessis, F. Fioranelli, H. Griffiths, M. R. Inggs, D. Jordan, W. Miceli, D. O'Hagan, R. Palama, S. Paine, M. Ritchie, S. J. Sandenbergh and A. Stevens, "First measurements with NeXtRAD, a polarimetric X/L Band radar network," in 2017 IEEE Radar Conference (RadarConf), Seattle, USA, 2017.

[14] S. Rahman and D. A. Robertson, "Millimeter-wave micro-Doppler measurements of small UAVs," in Radar Sensor Technology XXI, Anaheim, California, USA, 2017.

[15] M. R. Bell and R. A. Grubbs, "JEM modeling and measurement for radar target identification," IEEE Transactions on Aerospace and Electronic Systems, vol. 29, no. 1, pp. 73-87, 1993. 